Radiologe 2011 · 51:345

DOI 10.1007/s00117-011-2157-3

Online publiziert: 14. April 2011

(c) Springer-Verlag 2011
F. Bamberg $\cdot$ M. Reiser

Institut für Klinische Radiologie, Klinikum der Ludwig-Maximilians-Universität, Campus Großhadern, München

\title{
Radiologie im Zeitalter der Adipositas
}

\section{Implikationen, Herausforderungen und Chancen}

radiologischen Aspekte dieser beängstigenden demographischen Entwicklung zu geben. Die Kollegen Dr. Slawik und Prof. Beuschlein geben einen Überblick über die aktuelle klinisch-internistische Perspektive der Fettleibigkeit mit möglichen Behandlungsoptionen. Aus chirurgischer Sicht werden die neuesten bariatrischen Möglichkeiten und Verfahren erläutert und Komplikationen aus radiologischer Sicht veranschaulicht. Bei der CTDiagnostik, sicherlich dem Verfahren der Wahl, wird insbesondere der Dosisaspekt herausgearbeitet. Wir freuen uns auch, Ihnen einen innovativen Betrag aus Boston zur Wertigkeit der nichtinvasiven Fettgewebebestimmung mit CT und MRT für die klinische Praxis und die zukünftige Forschung vorzustellen. Exemplarisch wird außerdem am Beispiel der mit der Universität Greifswald assoziierten großen deutschen SHIP-Kohortenstudie verdeutlicht, wie die MRT eingesetzt werden könnte, um demographische Trends und Risikokonstellationen auch bei nichtadipösen asymptomatischen Probanden frühzeitig zu erkennen. In einem Ausblick schildern uns Dr. Sztrókay und PD Dr. Meindl, welchen potenziellen wissenschaftlichen Stellenwert die funktionelle MRT bei der Identifikation neuropsychologischer Ursachen der Fettsucht bietet. Insgesamt wird eindrucksvoll gezeigt, wie umfangreich die Radiologie sich dieser neuen Entwicklung angenommen hat und ihren Beitrag zur klinischen Versorgung wie auch zum wissenschaftlichen Erkenntnisgewinn auf vielfältige Weise leistet.
Wir hoffen, mit diesem Themenheft einen verständlichen und für die klinische Praxis nützlichen Überblick über den Stand der Entwicklung auf dem Gebiet der Adipositas zu geben und Ihr Interesse an diesem spannenden Thema zu wecken. Wir wünschen Ihnen viel Freude beim Lesen.

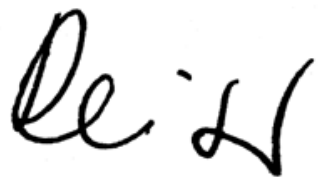

Prof. Dr. Maximilian Reiser

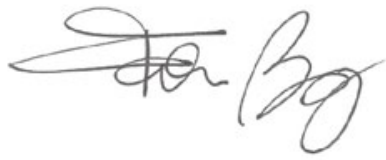

Dr. Fabian Bamberg

\section{Literatur \\ 1. Finucane MM, Stevens GA, Cowan MJ et al. on be- half of the Global Burden of Metabolic Risk Factors of Chronic Diseases Collaborating Group (Body Mass Index) (2011) National, regional, and global trends in body mass index since 1980: systematic analysis of health examination surveys and epide- miological studies with 960 country-years and 9,1 million participants. Lancet 2011 Feb 3. [Epub ahe- ad of print]}

